This item is the archived peer-reviewed author-version of:

Low-temperature chemical synthesis of high-purity diacylglycerols (DAG) from monoacylglycerols (MAG)

\title{
Reference:
}

Pazdur Lukasz, Geuens Jeroen, Sels Hannes, Tavernier Serge.- Low-temperature chemical synthesis of high-purity diacylglycerols (DAG) from monoacylglycerols (MAG)

Lipids / American Oil Chemist's Society - ISSN 1558-9307 - (2014), p. 1-8

Full text (Publishers DOI): http://dx.doi.org/doi:10.1007/s11745-014-3980-x

Handle/Permalink: http://hdl.handle.net/10067/1219910151162165141 


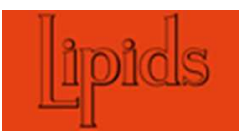

\section{Low-temperature chemical synthesis of high-purity diacylglycerols (DAG) from monoacylglycerols (MAG)}

\begin{tabular}{|r|l|}
\hline Journal: & Lipids \\
\hline Manuscript ID: & LIPIDS-14-0170.R2 \\
\hline Manuscript Type: & Methods \\
\hline Complete List of Authors: & $\begin{array}{l}\text { Pazdur, Lukasz; University of Antwerp, Faculty of Applied Engineering } \\
\text { Geuens, Jeroen; Karel de Grote University College, Dept. of Industrial } \\
\text { Sciences and Technology } \\
\text { Sels, Hannes; Karel de Grote University College, Dept. of Industrial } \\
\text { Sciences and Technology } \\
\text { Tavernier, Serge; University of Antwerp, Faculty of Applied Engineering }\end{array}$ \\
\hline Keywords: & $\begin{array}{l}\text { Lipid chemistry < General Area, Dietary fat < Nutrition, Diglyceride } \\
\text { synthesis < Specific Lipids }\end{array}$ \\
\hline
\end{tabular}


1

2

3

4

5

6

7

8

9

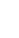

\section{Low-temperature chemical synthesis of high-purity diacylglycerols (DAG) from monoacylglycerols (MAG)}

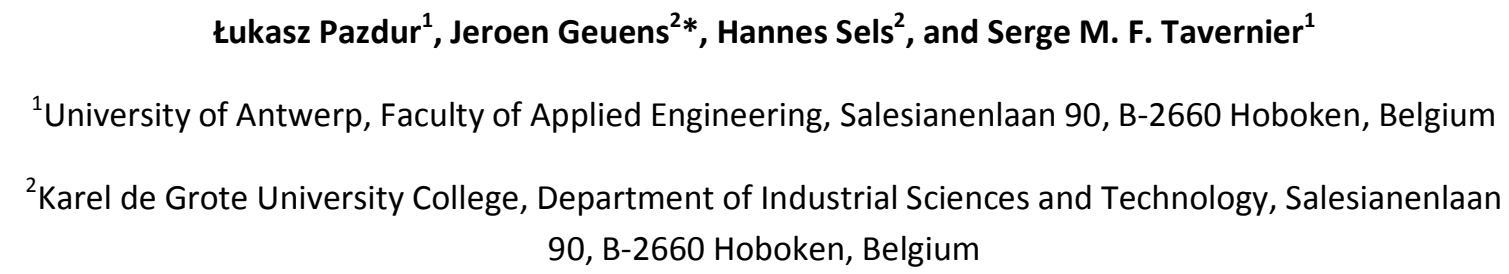

Abstract A chemical method was developed for low-temperature synthesis of DAG from MAG followed by an easy purification procedure in order to obtain high-purity DAG. Solvent-assisted and solvent-free reaction conditions were used, combined with different catalysts (sodium methoxide, ptoluenesulfonic acid, methanesulfonic acid, and sulfuric acid). All reactions were performed at 35 and $70^{\circ} \mathrm{C}$. By increasing both acidity and polarity of the catalyst the equilibrium shifts towards the formation of DAG. When using sulfuric acid in solvent-assisted condition at $70^{\circ} \mathrm{C}, 88 \%$ conversion was obtained after 20 min of reaction (77\% w/w DAG in the reaction mixture after evaporation of the solvent). After purifying by means of column chromatography, $96 \%$ pure DAG were obtained. The overall yield of DAG was $81 \%$.

Key words high-purity diacylglycerols (DAG), monoacylglycerols (MAG), transesterification, solventfree, solvent-assisted

\section{Abbreviations and symbols}

DAG Diacylglycerol(s)

FFA Free fatty acid(s)

GPC Gel permeation chromatography

MAG Monoacylglycerol(s) 
PTSA p-Toluenesulfonic acid

27 SA Solvent-assisted

28 SF Solvent-free

29 SFA Sulfuric acid

30 TAG Triacylglycerol(s)

31 TLC Thin layer chromatography

\section{Introduction}

Monoacylglycerols (MAG) and diacylglycerols (DAG) are widely used as emulsifiers in the food, cosmetic, pharmaceutical and chemical industry [1-3]. Recently DAG caught a lot of attention due to their nutritional properties and dietary effects [1,2,4-6]. Oils with a high DAG content are 'generally recognized as safe (GRAS)' [7] and have been commercialized in the USA [4,6,8] and Japan [4,6,9].

Diacylglycerols can be used to improve the quality of meat [10] or supress crystallization in order to reduce the solid fat content in palm oil [11]. Pure DAG can be used as building blocks for fatty acid derivatives [12] and as starting materials for synthesizing pharmaceutical products [13]. Because traditional chemical methods require high temperatures $[1,2,4,6,14]$ which cause auto-oxidation of unsaturated fatty acids [15], DAG are mainly produced by using enzyme catalysis $[1,2,4,9]$. Enzymatic production allows more selective production of DAG, resulting in low TAG content [16] while chemical approaches can lead to unwanted TAG (Fig. 1).

Some publications address low-temperature, non-enzymatic synthesis of DAG from MAG $[17,18]$, however, the methods, which these publications refer to, are not suitable for large-scale production of DAG. Isai et al used 1:640 (w/w) MAG/solvent ratio and 5-10\% (w/w) p-toluenesulfonic acid catalyst (based on MAG mass), yielding $12.8 * 10^{-6} \mathrm{M}$ concentration of DAG [17]. The synthesis by 
Stamatov et al was intended to produce enantiomerically pure products and involved three-step synthesis [18].

In the present work we developed a low-temperature highly selective chemical method to produce DAG from MAG followed by an easy purification step, which yielded $96 \%$ pure DAG. The method can easily be scaled up to produce large quantities of pure DAG.

\section{Materials and methods}

\section{Materials}

Monoacylglycerols were donated by Oleon NV (Oelegem, Belgium), they contained 95.1\% MAG, 3.8\% DAG, $0.3 \%$ free glycerol and the acid value was $1.6 \mathrm{mg} \mathrm{KOH} / \mathrm{g}$. Table 1 shows the fatty acid profile of this product. Sodium methoxide ( $98 \%$ purity) was obtained from Alfa Aesar (Karlsruhe, Germany). pToluenesulfonic acid monohydrate ('for analysis' grade) was obtained from Merck Chemicals (Darmstadt, Germany). Methanesulfonic acid (99\%), sulphuric acid (96\%), acetic acid (100\%), ethyl acetate, methanol $(99 \%)$, tetrahydrofuran $(99 \%)$, and petroleum ether $\left(40-60{ }^{\circ} \mathrm{C}\right)$ were analytical grade and obtained from Acros Organics (Geel, Belgium). Orcinol (98\%) and silica gel for chromatography were obtained from Acros Organics.

\section{Reaction Procedures}

Diacylglycerols were formed through intermolecular exchange of acyl moieties in MAG molecules.

Transesterification between two MAG results in the formation of one DAG and one glycerol molecule (see Fig. 1a). The reaction was performed both acid- and base-catalyzed, and all reactions were performed at 35 and $70^{\circ} \mathrm{C}$. To shift the equilibrium and increase the DAG yield, petroleum ether was added to the reaction mixture as solvent. Monoacylglycerols were dried under vacuum before use to prevent reaction between water and the sodium methoxide catalyst. All reactions were performed in a three-necked glass flask ( $250 \mathrm{~mL}$ ) equipped with a cooler and placed in an oil bath held at constant temperature. The reaction mixture was stirred with a magnetic stirrer. All reactions were performed in duplicate. 
Sodium methoxide is a good catalyst to perform transesterifications [19]. Thus, as a reference, the reaction was performed by using sodium methoxide at 35 and $70{ }^{\circ} \mathrm{C}$ under both solvent-free and solvent-assisted conditions. For solvent-free reactions, $0.033 \mathrm{~mol}$ of sodium methoxide per mol MAG was added after heating the MAG. For solvent-assisted reactions, the MAG were mixed with petroleum ether in 1:1 mass ratio. This mixture was heated to the desired reaction temperature before the catalyst was added.

For acid-catalyzed solvent-free reactions, $0.088 \mathrm{~mol} / \mathrm{mol}$ of $\mathrm{p}$-toluenesulfonic acid (dissolved in 1.5 $\mathrm{mL}$ tetrahydrofuran to increase the solubility and prevent the rate of dissolution to be the limiting factor in reaction kinetics), methanesulfonic acid or sulfuric acid were added after heating the MAG. Samples $(5 \mathrm{~mL})$ were collected during the first $60 \mathrm{~min}$ of reaction and were immediately treated with 2-3 drops of a $7 \mathrm{M}$ potassium carbonate solution to neutralize the catalyst. For solvent-assisted reactions, the same procedure was followed but the MAG were mixed with the solvent in 1:1 mass ratio before heating.

\section{Analysis of the Reaction Mixture}

The final reaction mixtures as well as intermediate samples were analyzed by means of gel permeation chromatography (GPC) by using an Agilent 1100 series system (Diegem, Belgium) and isocratic elution with THF on a $300 \mathrm{~mm} \times 7.80 \mathrm{~mm}, 5$ micron, $100 \AA$ Phenogel (Phenomenex, Utrecht, The Netherlands) column connected to a refractive index detector. The common errors for the determination of the amounts of MAG, DAG, and TAG were 1-2\% (w/w).

\section{Purification}

The reaction mixture was purified by means of column chromatography $(500 \mathrm{~mm} \times 50 \mathrm{~mm}$ glass column) on silicagel (60-200 $\mu \mathrm{m}, 60 \AA$ ) by using the following analytical-grade solvents: petroleum ether, ethyl acetate, acetic acid, and methanol [20]. Silica gel was mixed with an appropriate amount of petroleum ether and packed into the column (approx. $350 \mathrm{~mm}$ of stationary phase). 
97 About $20 \mathrm{~g}$ of raw mixture was dissolved in $20 \mathrm{~g}$ of petroleum ether and loaded on the column.

98 Samples were eluted with different solvent mixtures [20] (see Table 2). The amount of solvent was

99 slightly reduced compared to Zhao et al [20]. Samples (30 mL) were collected and analyzed by TLC.

100 Each of the collected samples was spotted on a TLC plate for qualitative analysis. On each TLC plate a

101 sample of crude reaction mixture disolved in petroleum ether was spotted as a reference. Some

102 fractions were injected on GPC for identification of the spots.TLC (silica gel 60W plate) was

103 performed by using 90:10:1 (v/v) petroleum ether/ethyl acetate/acetic acid as the mobile phase.

104 Visualization was carried out by spraying orcinol solution ( $0.1 \% 5$-methylresorcinol dissolved in $5 \%$

$105 \mathrm{H}_{2} \mathrm{SO}_{4}$ ) on the TLC-plate and heating it at $110{ }^{\circ} \mathrm{C}$ for $10 \mathrm{~min}$ [21].

106 After TLC analysis the samples containing the same components were combined and after

107 evaporation of the solvent, the pure products were obtained. Pure products were analyzed on GPC

108 to determine the purity of the obtained products.

\section{Results}

Transesterification Reactions of MAG

\section{Base-catalyzed Reactions}

112 Sodium methoxide is a good catalyst for transesterification [19], thus, reference experiments with

113 sodium methoxide were performed for $60 \mathrm{~min}$ at 35 and $70{ }^{\circ} \mathrm{C}$ using solvent-free and solvent-

114 assisted conditions. The grade of conversion of MAG to DAG was calculated according to Eq. 1.

115 Conversion $(\%)=\frac{\text { Actual amount of DAG }(\% \mathrm{w} / \mathrm{w})}{\text { Theoretical maximum amount of DAG }(\% \mathrm{w} / \mathrm{w})} \times 100 \% \quad$ [Eq. 1]

116 Whereas the theoretical maximum amount of DAG is calculated as follows:

117 Theoretical maximum amount of DAG $(\% \mathrm{w} / \mathrm{w})=\frac{\left(\mathrm{m}_{\mathrm{MAG}} / \mathrm{MM}_{\mathrm{MAG}}\right) \times \mathrm{MM}_{\mathrm{DAG}}}{\mathrm{m}_{\text {reaction mixture }}}$ [Eq. 2]

$118 \mathrm{~m}_{\mathrm{MAG}}=$ mass of $\mathrm{MAG}$ added to the reaction mixture $(\mathrm{g})$

$119 \mathrm{MM}_{\mathrm{MAG}}=$ molar mass of $\mathrm{MAG}(\mathrm{g} / \mathrm{mol})$ 
$\mathrm{MM}_{\mathrm{DAG}}=$ molar mass of DAG $(\mathrm{g} / \mathrm{mol})$

121

$m_{\text {reaction mixture }}=$ total mass of the reaction mixture $(\mathrm{g})$

122
The equilibrium conversion was determined by plotting conversion (Eq. 1) versus time and by nonlinear fitting of the data by using the Prism 6 software from Graphpad Software Inc (La Jolla, California, USA). The equilibrium amounts were the plateau values of the fitted curves. For alkalicatalyzed solvent-free reaction at $70^{\circ} \mathrm{C}$, the equilibrium conversion, being $48 \pm 1 \%$, was reached after 10 min while the reaction at $35^{\circ} \mathrm{C}$ reached an equilibrium conversion of $47 \pm 1 \%$ after $30 \mathrm{~min}$.

In order to obtain a higher yield of DAG, reactions were performed in the presence of petroleum ether using a 1:1 mass ratio MAG/petroleum ether. Since petroleum ether is a nonpolar solvent, it expelled the glycerol from the glyceride phase, thereby shifting the equilibrium towards DAG formation (Fig. 1a). For solvent-assisted base-catalyzed reaction at $70{ }^{\circ} \mathrm{C}$, the equilibrium conversion, being $65 \pm 1 \%$, was reached after $15 \mathrm{~min}$; at $35^{\circ} \mathrm{C}$, an equilibrium conversion of $64 \pm 1 \%$ was reached after $50 \mathrm{~min}$. When these data are compared to the analogue solvent-free reaction, the equilibrium conversion increased by $17 \%$ when petroleum ether is used as a solvent.

Fig. 2 shows the concentrations of MAG, DAG and TAG in the reaction mixture for all reactions performed with sodium methoxide as a catalyst. The highest amount of DAG was obtained at $70{ }^{\circ} \mathrm{C}$ by using petroleum ether as solvent. However, when considering the TAG content, the highest amount of TAG was also observed at these conditions.

\section{Solvent-free Acid-catalyzed Reactions}

Three acidic catalysts were used to convert MAG to DAG, p-toluenesulfonic acid, methanesulfonic acid and sulfuric acid. Fig. 3 shows the amount of DAG versus time for all experiments. For the reaction with p-toluenesulfonic acid at $70{ }^{\circ} \mathrm{C}$, the equilibrium was achieved after 15 min while at 35 ${ }^{\circ} \mathrm{C}$, equilibrium was not achieved within $60 \mathrm{~min}$. However, when prolonging the reaction time, equilibrium was reached after $150 \mathrm{~min}$. The equilibrium conversions were $53 \pm 1 \%$ at $35{ }^{\circ} \mathrm{C}$ and $56 \pm$ 
$1441 \%$ at $70{ }^{\circ} \mathrm{C}$, both higher than the conversion which was achieved when using sodium methoxide as 145 catalyst. Using methanesulfonic acid, at $70^{\circ} \mathrm{C}$, equilibrium was reached within 15 min while at $35^{\circ} \mathrm{C}$, 146 it took $30 \mathrm{~min}$ to reach equilibrium. The equilibrium conversions were $74 \pm 3 \%$ at $35^{\circ} \mathrm{C}$ and $73 \pm 1 \%$ at

$14770^{\circ} \mathrm{C}$, which were higher than conversions reached when using sodium methoxide or $\mathrm{p}$ -

148 toluenesulfonic acid. With sulfuric acid as a catalyst equilibrium was reached after 15 min at $35^{\circ} \mathrm{C}$,

149 while at $70{ }^{\circ} \mathrm{C}$, equilibrium was reached after $10 \mathrm{~min}$. The equilibrium conversions were $73 \pm 2 \%$ at 35

$150{ }^{\circ} \mathrm{C}$ and $75 \pm 2 \%$ at $70{ }^{\circ} \mathrm{C}$ which were in the same conversion range as when using methanesulfonic 151 acid. Fig. 4 summarizes the results for all alkali- and acid-catalyzed solvent-free reactions. It can be 152 seen that in alkali-catalyzed conditions, significantly more unwanted TAG are produced.

153 In order to determine the effects of catalyst concentration on reaction kinetics, the amount of 154 catalyst was reduced from 0.088 to 0.044 and $0.022 \mathrm{~mol} / \mathrm{mol}$. Lower concentration of catalyst 155 increases the time to reach equilibrium (Fig. 5). The amount of catalyst altered the equilibrium conversion: $73 \pm 1 \%, 70 \pm 1 \%$ and $65 \pm 2 \%$ conversion were reached using $0.088,0.044$ and 0.022 $\mathrm{mol} / \mathrm{mol}$ methanesulfonic acid, respectively. This phenomenon is discussed further on (see Discussion - Comparison of the Reaction Equilibrium for the Acid-catalyzed Reactions). Solvent-assisted Acid-catalyzed Reactions

The acidic catalysts used in solvent-free conditions were also tested in solvent-assisted reactions. Fig. 6 shows the amount of DAG versus time for all experiments. When p-toluenesulfonic acid was used as catalyst, after $20 \mathrm{~min}$ of reaction at $70{ }^{\circ} \mathrm{C}, 82 \pm 2 \%$ conversion was obtained. For reaction at $35^{\circ} \mathrm{C}$ using the same catalyst, equilibrium was not reached within $60 \mathrm{~min}$ and even when reaction time was extended to $300 \mathrm{~min}$, equilibrium could not be reached. When comparing the results of the solvent-assisted reactions with those of the solvent-free reactions, conversion was $26 \%$ higher when petroleum ether was used. For reaction with methanesulfonic acid at $70{ }^{\circ} \mathrm{C}, 85 \pm 1 \%$ conversion was obtained after $10 \mathrm{~min}$ of reaction while at $35^{\circ} \mathrm{C}$, it took $30 \mathrm{~min}$ to reach $82 \pm 2 \%$ conversion. Once again, 8 to $12 \%$ increase in conversion was achieved when petroleum ether was added as solvent. The last acid-catalyzed solvent-assisted reactions were performed using sulfuric acid as catalyst. 
Equilibrium was achieved after $20 \mathrm{~min}$ at $70^{\circ} \mathrm{C}$, while at $35^{\circ} \mathrm{C}$, it took $50 \mathrm{~min}$ to achieve equilibrium.

171 The equilibrium conversion at $70^{\circ} \mathrm{C}$ was $88 \pm 1 \%$ while at $35^{\circ} \mathrm{C}$, the equilibrium conversion was $82 \pm$

$1722 \%$. Fig. 7 summarizes the results for all solvent-assisted reactions. Once again, it can be seen that

173 more unwanted TAG are produced in base-catalyzed conditions. When comparing solvent-free and

174 solvent-assisted reactions (see Fig. 4 and Fig. 7), one can observe that the time to reach equilibrium

175 slightly increases when a solvent is added. However, conversion, and thus also the amount of DAG,

176 significantly increases in solvent-assisted conditions.

177 Purification of the Reaction Mixture

178 The crude reaction mixture contains TAG, DAG, MAG, glycerol, catalyst and traces of FFA. Phase

179 separation was observed in all reactions. The glycerol phase contained the polar compounds (glycerol

180 and catalyst), the glyceride phase contained the solvent (if added) and the glycerides. The literature

181 describes several methods to purify mixtures of different fatty acid compounds: liquid-liquid

182 extraction [23], molecular distillation $[14,24]$ and column chromatography $[11,20]$.

183 Two techniques were applied to obtain high-purity DAG: liquid-liquid extraction and column

184 chromatography. Simple liquid-liquid extraction with water removed glycerol and catalyst from the

185 crude mixture. The second step in this procedure aims to selectively extract the DAG from the

186 glyceride phase [23]. Several solvent mixtures were tested but gave unfavourable results in

187 separating the different glycerides. Hence, column chromatography was the most suitable technique.

188 Following the procedure mentioned earlier (see Experimental Procedures - Purification), about 70

189 samples were collected from the column. The TAG eluted first and were observed in fractions 8 to

190 24. Subsequently, DAG eluted (fractions 27 to 55) with the last part of eluent I (see Table 2),

191 however, the highest concentration of DAG was observed in the fractions containing eluent II.

192 Fractions 56 and higher contained the MAG and glycerol. The MAG were eluted with eluents III to V.

193 Glycerol was eluted with methanol. 
The DAG were $96 \%$ pure (impurities mainly MAG) and were obtained at $92 \%$ recovery. Taking into account $88 \%$ conversion (Fig. 7 ) this results in $81 \%$ overall yield of high-purity DAG. Thus, preparative liquid chromatography or moving bed chromatography are promising possibilities for isolating DAG on industrial scale.

Discussion

\section{Comparison of the Reaction Equilibrium for the Acid-catalyzed Reactions}

Fig. 8 shows the conversions for all three acidic catalysts when performing solvent-free or solventassisted reactions at 35 or $70^{\circ} \mathrm{C}$. It can be seen that other degrees of conversion were reached when different catalysts were used. This phenomenon can be explained by the fact that during reaction, two phases were formed: a glycerol phase and a glyceride phase. A polar catalyst will go to the glycerol phase, thereby increasing the polarity of this phase (Fig. 9). When the polarity of the glycerol phase increases, more glycerol is attracted to this phase shifting the equilibrium toward DAG formation (Fig. 1a). This hypothesis was confirmed by measuring the amount of glycerol and sulfur in the glyceride phase after conducting solvent-free reactions at $35^{\circ} \mathrm{C}$ with different acidic catalysts. The reaction was carried out until equilibrium conversion was reached. Hence, reaction time depended on the catalyst used, thus the reaction time was 180 min for p-toluenesulfonic acid, while it was 60 min for methanesulfonic acid and sulfuric acid. Samples were taken after these reaction times. The catalyst was not neutralized and the sample was centrifuged at $35^{\circ} \mathrm{C}$ for $30 \mathrm{~min}$. The sulfur content and the glycerol content in the glyceride phase were measured, respectively by means of XRF and GPC. From the results shown in Table 3, it can be seen that p-toluenesulfonic acid, being the most apolar of the three catalysts, gave the highest glycerol content and the highest sulfur content in the glyceride phase. Sulfuric acid, the most polar of the three catalysts, yielded no detectable glycerol in the glyceride phase and had the lowest sulfur concentration in said layer. Thus, GPC and XRF analyses confirmed that a more polar catalyst goes into the glycerol phase, leading to a more polar glycerol phase, less glycerol in the glyceride phase, and thus to higher conversion. This 
different concentrations (see Fig. 5). Higher concentration of catalyst cause the glycerol phase to be

221

222 more polar and induces higher conversions.

Extra experiments were conducted to determine the relationship between catalyst acidity and conversion. Reactions were performed at $70{ }^{\circ} \mathrm{C}$ for $60 \mathrm{~min}$ in solvent-free conditions using 0.088 $\mathrm{mol} / \mathrm{mol}$ acetic acid, phosphoric acid, or trichloroacetic acid as catalyst. In Fig. 10 the results of these reactions are compared with those of reactions with p-toluenesulfonic acid, methanesulfonic acid, and sulfuric acid (pKa values from reference 20). pKa values smaller than zero were needed to obtain $>50 \%$ conversion.

\section{Comparison of the Reaction Kinetics for the Acid-catalyzed Reactions}

In order to compare the kinetics of the different acid-catalyzed reactions, the amount of MAG was plotted versus reaction time and a nonlinear fit of these data was constructed. The general equation of these curves is shown in Eq. 3.

$\mathrm{n}=\mathrm{n}_{0}+\left(\mathrm{n}_{\mathrm{eq}}-\mathrm{n}_{0}\right) \cdot\left(1-\mathrm{e}^{-\mathrm{kt}}\right) \quad$ [Eq. 3]

$\mathrm{n}=$ molar amount at time $\mathrm{t}\left[\mathrm{mol}^{*} 10^{-2}\right]$

$\mathrm{n}_{0}=$ molar amount at time zero $\left[\mathrm{mol}^{*} 10^{-2}\right]$

$\mathrm{n}_{\mathrm{eq}}=$ equilibrium amount $\left[\mathrm{mol}^{*} 10^{-2}\right]$

$\mathrm{k}=$ rate constant $\left[\mathrm{min}^{-1}\right]$

$\mathrm{t}=$ time $[\mathrm{min}]$

From Eq. 3, half-time values $\left(t_{1 / 2}\right)$ can be calculated, which is the time for MAG to reach $50 \%$ of the original amount $\left(\mathrm{n}_{0}\right)$. Half-time values give good indications about reaction kinetics. The half-time value of MAG and the correlation coefficient of the fitted curves are given in Table 4. As expected, reactions at $70{ }^{\circ} \mathrm{C}$ had lower half-time values than those conducted at $35^{\circ} \mathrm{C}$. The solvent-assisted reactions were slower than the solvent-free reactions. This is due to a more efficient extraction of the catalyst to the glycerol phase in solvent-assisted conditions and dilution of the catalyst when adding petroleum ether. 
Transesterification can be carried out both in base- and acid-catalyzed modes. However, unwanted

formation of TAG is suppressed in acid-catalyzed conditions. In order to reach high equilibrium amounts of DAG, the $\mathrm{pK}_{\mathrm{a}}$ value of the acidic catalyst should be $<0$. The polarity of the catalyst also plays an important role: more polar catalysts move to the polar glycerol phase during reaction, thereby increasing the polarity of the glycerol phase and shifting the equilibrium in favour of DAG production. Consequently, higher conversions are achieved with more polar catalysts. Although acceptable conversion was achieved in solvent-free reactions, conversions can be increased by using a solvent. The solvent petroleum ether can easily be distilled and reused because of its low boiling point. Solvent-assisted reactions have slower kinetics, but equilibrium is still reached after 20 min. Maximum conversion (88\%) was achieved in sulfuric acid-catalyzed, solvent-assisted reaction at 70 of $81 \%$ was achieved. The methods presented in this work are suitable for large-scale production of high-purity DAG. Although higher conversions are reached when working with petroleum ether as solvent, this method is less environmentally benign. Moreover, the overall cost of the process will increase when a solvent is used. Thus, both economic and environmental parameters will need to be evaluated in order to choose the right process for large-scale production.

\section{Acknowledgements}

The authors thank Karel de Grote-Hogeschool for financial support and Oleon NV for providing the MAG.

\section{References}

[1] Feltes MMC, de Oliveira D, Block JM, Ninow JL (2013) The Production, Benefits, and Applicationsof Monoacylglycerols and Diacylglycerols of Nutritional Interest. Food Bioprocess

[2] Hamerski F, Corazza ML (2014) LDH-catalyzed esterification of lauric acid with glycerol in solvent-free system. Appl Catal A - Gen 475:242-248 
271 [3] Dijkstra AJ (2007) Food grade emulsifiers. In: Gunstone FD, Harwood JL, Dijkstra AJ (eds) The 272 Lipid Handbook, 3rd edn. CRC Press, Boca Raton, pp 315-333

273 [4] Zhong N, Deng X, Huang J, Xu L, Hu K, Gao Y (2014) Low-temperature chemical glycerolysis to 274 produce diacylglycerols by heterogeneous base catalyst. Eur J Lipid Sci Technol 116:470-476

275 [5] Lo S-K, Tan C-P, Long K, Yusoff MSA, Lai O-M (2008) Diacylglycerol oil-Properties, processes and 276 products: a review. Food Bioprocess Technol 1:223-233

277 [6] Zhong N, Li L, Xu X, Cheong L-Z, Zhao X, Li B (2010) Production of diacylglycerols through low-

278 temperature chemical glycerolysis. Food Chem 122:228-232

279 [7] Morita O, Soni MG (2009) Safety assessment of diacylglycerol oil as an edible oil: A review of the published literature. Food Chem Toxicol 47:9-21

[8] Caravan Ingredients (accessed June 2014) Trancendim. http://www.caravaningredients.com/brands/trancendim.aspx

[9] Kao (accessed June 2014) Research and Development of Diacylglycerol. http://www.kao.co.jp/rd/dag_en/index.html

[10] Miklos R, Xu X, Lametsch R (2011) Application of pork fat diacylglycerols in meat emulsions. Meat Sci 87:202-205

[11] Siew W-L, Ng W-L (1999) Influence of diglycerides on crystallization of palm oil. J Sci Food Agric 79:722-726

[12] Haftendorn R, Ulbrich-Hofmann R (1995) Synthesis of 2-modified 1,3-diacylglycerols. Tetrahedron 51:1177-1186 
291

292

293

294

295

296

297

298

299

300

301

302

303

304

305

306

307

308

309

310

311

312

[13] Vodovozova EL, Gaenko GP, Bobrikova ES, Pazynina GV, Molotkovskii YG (2007) A diglyceride derivative of methotrexate: synthesis and cytotoxic activity in addressed liposomes. Pharm Chem J 41:297-301

[14] Jacobs L, Lee I, Poppe G (2006) Chemical process for the production of 1,3-diglyceride oils. U.S. Patent no. 7081542

[15] Park J-Y, Kim D-K, Lee J-P, Park S-C, Kim Y-J, Lee J-S (2008) Blending effects of biodiesels on oxidation stability and low temperature flow properties. Bioresource Technol 99:1196-1203

[16] Guo Z, Sun Y (2007) Solvent-free production of 1,3-diglyceride of CLA: Strategy consideration and protocol design. Food Chem 100:1076-1084

[17] Isai SV, Usol'tsev AA, Stiba EN (2003) Acid-catalyzed intra- and intermolecular acyl exchange in mono- and diglycerides. Chem Nat Compd 39:325-329

[18] Stamatov SD, Stawinski J (2007) Regioselective and stereospecific acylation across oxirane- and silyloxy systems as a novel strategy to the synthesis of enantiomerically pure mono-, di- and triglycerides. Org Biomol Chem 5:3787-3800

[19] Atadashi IM, Aroua MK, Aziz ARA, Sulaiman NMN (2013) The effects of catalysts in biodiesel production: A review. J Ind Eng Chem 19:14-26

[20] Zhao Y, Liu J, Deng L, Wang F, Tan T (2011) Optimization of Candida sp. 99-125 lipase catalyzed esterification for synthesis of monoglyceride and diglyceride in solvent-free system. J Mol Catal B - Enzym 72:157-162

[21] Onghena M, Geens T, Goossens E, Wijnants M, Pico Y, Neels H, Covaci A, Lemiere F (2011) Analytical characterization of mannosylerythritol lipid biosurfactants produced by biosynthesis based on feedstock sources from the agrofood industry. Anal Bioanal Chem 400:1263-1275 
313 [22] Wade LG Jr. (2013) Organic Chemistry, 8th international edn. Pearson, Glenview, p 1257

314 [23] Peter S, Czech B, Ender U, Weidner E (1995) Process for preparing pure monoglycerides, pure 315 diglycerides and/or pure triglycerides. U.S. Patent no. 5434280

316 [24] Wang Y, Zhao M, Song K, Wang L, Han X, Tang S, Wang Y (2010) Separation of diacylglycerols

317 from enzymatically hydrolyzed soybean oil by molecular distillation. Sep Purif Technol 75:114-

$318 \quad 120$

319 
Fig. 1 (a) transesterification of 2 MAG molecules, (b) transesterification of a MAG and a DAG molecule, (c) transesterification of a MAG and a TAG molecule

322

Fig. 2 Base-catalyzed transesterification of MAG using sodium methoxide catalyst, concentration of MAG, DAG and TAG in the glyceride phase after $60 \mathrm{~min}$ of reaction (for solvent-assisted reactions, petroleum ether was evaporated before GPC measurement)

Fig. 3 DAG concentration versus time for solvent-free transesterification of MAG at (a) $35^{\circ} \mathrm{C}$ and (b) $70^{\circ} \mathrm{C}$ (PTSA=p-toluenesulfonic acid, MSA=methanesulfonic acid, SFA=sulfuric acid)

Fig. 4 Overview of the results for all solvent-free reactions $(S M O=$ sodium methoxide, PTSA=ptoluenesulfonic acid, MSA=methanesulfonic acid, SFA=sulfuric acid), ${ }^{*}$ for PTSA at $35^{\circ} \mathrm{C}$ the equilibrium time was $300 \mathrm{~min}$

Fig. 5 Solvent-free, acid-catalyzed transesterification of MAG using different amounts of catalyst (methanesulfonic acid, $70^{\circ} \mathrm{C}$ )

Fig. 6 DAG concentration versus time for solvent-assisted transesterification of MAG at (a) $35^{\circ} \mathrm{C}$ and (b) $70^{\circ} \mathrm{C}$ (PTSA=p-toluenesulfonic acid, MSA=methanesulfonic acid, SFA=sulfuric acid)

Fig. 7 Overview of the results for all solvent-assisted reactions ( $S M O=$ sodium methoxide, PTSA=ptoluenesulfonic acid, MSA=methanesulfonic acid, SFA=sulfuric acid), * for PTSA at $35^{\circ} \mathrm{C}$ reaction time was 300 min (equilibrium could not be reached)

Fig. 8 Conversion for synthesis of DAG from MAG at $70^{\circ} \mathrm{C}$ and $35^{\circ} \mathrm{C}$ in solvent-assisted (SA) and solvent-free (SF) conditions using different acidic catalysts (PTSA = p-toluenesulfonic acid, MSA = methanesulfonic acid, SFA = sulfuric acid), standard reaction time of $60 \mathrm{~min}$ (prolonged to $180 \mathrm{~min}$ for PTSA-SF in order to reached equilibrium, prolonged to $300 \mathrm{~min}$ for PTSA-SA without reaching equilibrium)

Fig. 9 Schematic representation of catalyst movement to glycerol layer for solvent-free and solventassisted reactions with (a) p-toluenesulfonic acid, (b) methanesulfonic acid and (c) sulfuric acid. Line in the middle represents the phase separation.

Fig. 10 Solvent-free acid-catalyzed transesterification of MAG using different acidic catalysts (60 min, $70^{\circ} \mathrm{C}$ ), pKa versus conversion ( $\mathrm{pKa}$ values from reference 22 ) 
Table 1 Fatty acid profile of starting material (MAG from Oleon)

\begin{tabular}{|l|c|}
\hline Fatty acid & $\%(\mathrm{w} / \mathrm{w})$ \\
\hline C16:0 & 7.9 \\
\hline C18:1t & 3.1 \\
\hline C18:1c & 35.2 \\
\hline C18:2 & 53.5 \\
\hline C18:3 & 0.3 \\
\hline
\end{tabular}

349

350 Table 2 Solvent mixtures applied for column elution with reduced solvent use

\begin{tabular}{ccc}
\hline Volume $(\mathrm{mL})$ & Solvents & Mixture $(\mathrm{v} / \mathrm{v})$ \\
\hline 1200 & Petroleum ether/ethyl acetate/acetic acid (I) & $90: 10: 1$ \\
300 & Petroleum ether/ethyl acetate/acetic acid (II) & $80: 20: 1$ \\
600 & Petroleum ether/ethyl acetate/acetic acid (III) & $70: 30: 0.7$ \\
300 & Petroleum ether/ethyl acetate/acetic acid (IV) & $50: 50: 1$ \\
100 & Ethyl acetate (V) & 100 \\
600 & Methanol (VI) & 100 \\
\hline
\end{tabular}

351

352

353

354

355

356

357

358

Table 4 Half time values [min] and correlation coefficients after nonlinear fit of amount of MAG (PTSA = p-toluenesulfonic acid, MSA = methanesulfonic acid, SFA = sulfuric acid)

\begin{tabular}{|c|c|c|c|c|c|c|c|c|}
\cline { 2 - 9 } \multicolumn{1}{c|}{} & \multicolumn{4}{c|}{$35^{\circ} \mathrm{C}$} & \multicolumn{4}{c|}{$70^{\circ} \mathrm{C}$} \\
\cline { 2 - 9 } \multicolumn{1}{c|}{} & \multicolumn{2}{c|}{ Solvent-free } & \multicolumn{2}{c|}{ Solvent-assisted } & \multicolumn{2}{c|}{ Solvent-free } & \multicolumn{2}{c|}{ Solvent-assisted } \\
\hline Catalyst & $\mathrm{t}_{1 / 2}$ & $\mathrm{R}^{2}$ & $\mathrm{t}_{1 / 2}$ & $\mathrm{R}^{2}$ & $\mathrm{t}_{1 / 2}$ & $\mathrm{R}^{2}$ & $\mathrm{t}_{1 / 2}$ & $\mathrm{R}^{2}$ \\
\hline PTSA & 30.00 & 0.9977 & 47.40 & 0.9904 & 3.13 & 0.9919 & 5.03 & 0.9926 \\
\hline MSA & 1.47 & 0.9587 & 3.06 & 0.9731 & 0.50 & 0.9965 & 0.56 & 0.9964 \\
\hline SFA & 0.99 & 0.9654 & 3.03 & 0.9603 & 0.45 & 0.9892 & 0.46 & 0.9883 \\
\hline
\end{tabular}

Table 3 Conversion and concentration of sulfur and glycerol in the glyceride layer after solvent-free reaction at $35^{\circ} \mathrm{C}$ using different acidic catalysts (PTSA $=p$-toluenesulfonic acid, MSA = methanesulfonic acid, SFA = sulfuric acid), samples were taken after equilibrium concentration was reached (180 min for PTSA, 60 min for MSA and SFA)

\begin{tabular}{|l|c|c|c|}
\cline { 2 - 4 } \multicolumn{1}{c|}{} & \multicolumn{2}{c|}{ Concentration in glyceride phase } & Conversion \\
\cline { 2 - 4 } \multicolumn{1}{c|}{} & Sulfur [ppm] & Glycerol [\% w/w] & {$[\%]$} \\
\hline PTSA & 4410 & 6.8 & 55 \\
\hline MSA & 1795 & 1.4 & 73 \\
\hline SFA & 65 & 0.0 & 77 \\
\hline
\end{tabular}


(a)

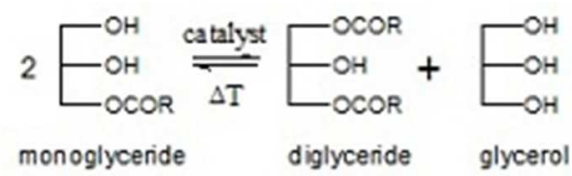

(b)

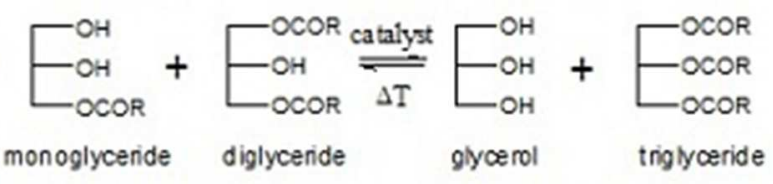

(c)

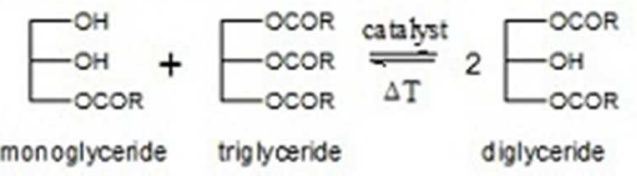

Fig. 1 (a) transesterification of 2 MAG molecules, (b) transesterification of a MAG and a DAG molecule, (c) transesterification of a MAG and a TAG molecule $85 \times 56 \mathrm{~mm}(96 \times 96$ DPI $)$ 


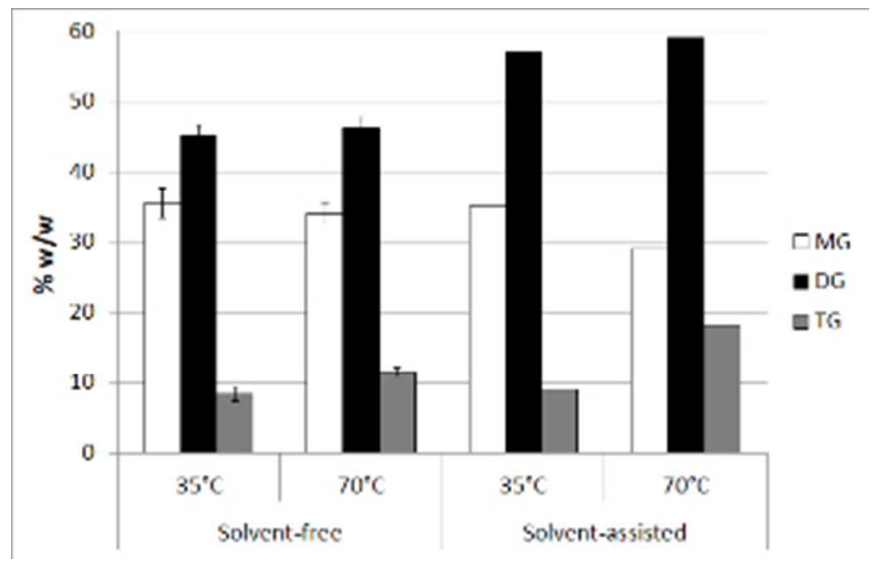

Fig. 2 Base-catalyzed transesterification of MAG using sodium methoxide catalyst, concentration of MAG, DAG and TAG in the glyceride phase after 60 min of reaction (for solvent-assisted reactions, petroleum ether was evaporated before GPC measurement)

$87 \times 55 \mathrm{~mm}$ (96 x 96 DPI) 

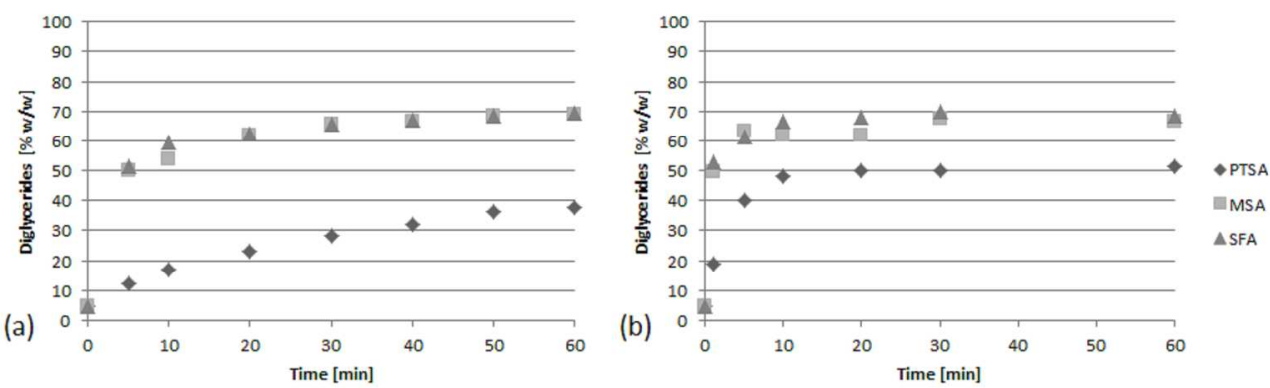

Fig. 3 DAG concentration versus time for solvent-free transesterification of MAG at (a) $35^{\circ} \mathrm{C}$ and (b) $70{ }^{\circ} \mathrm{C}$ (PTSA=p-toluenesulfonic acid, MSA=methanesulfonic acid, SFA=sulfuric acid) $485 \times 152 \mathrm{~mm}$ (96 x $96 \mathrm{DPI})$ 


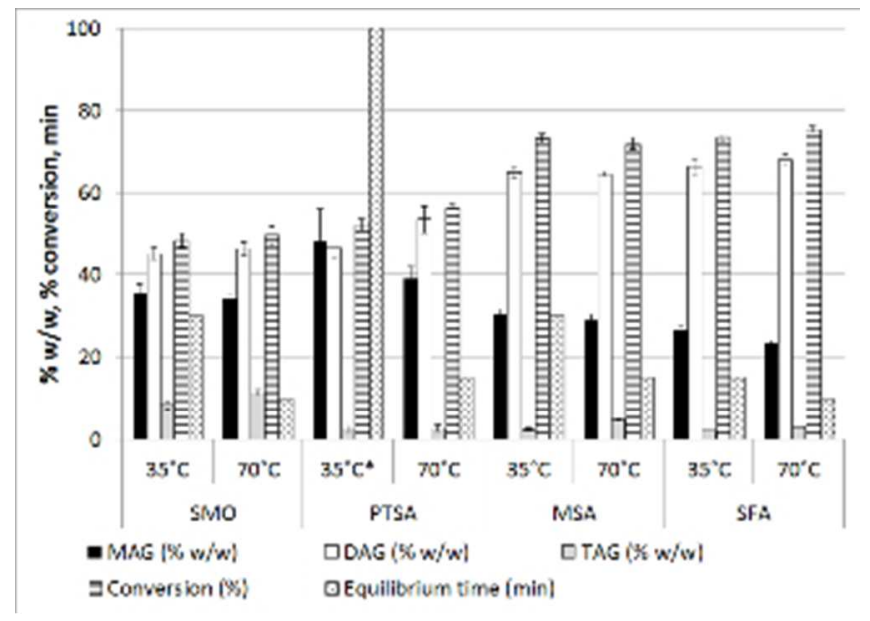

Fig. 4 Overview of the results for all solvent-free reactions $(S M O=$ sodium methoxide, PTSA=ptoluenesulfonic acid, MSA=methanesulfonic acid, SFA=sulfuric acid), * for PTSA at $35^{\circ} \mathrm{C}$ the equilibrium time was $300 \mathrm{~min}$ $85 \times 61 \mathrm{~mm}(96 \times 96 \mathrm{DPI})$ 


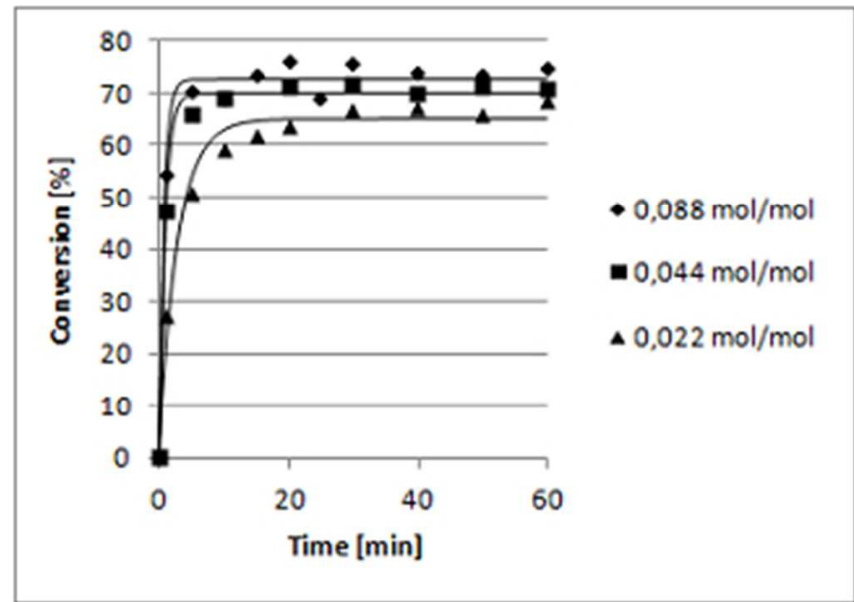

Fig. 5 Solvent-free, acid-catalyzed transesterification of MAG using different amounts of catalyst (methanesulfonic acid, $70^{\circ} \mathrm{C}$ ) $85 \times 61 \mathrm{~mm}(96 \times 96 \mathrm{DPI})$ 

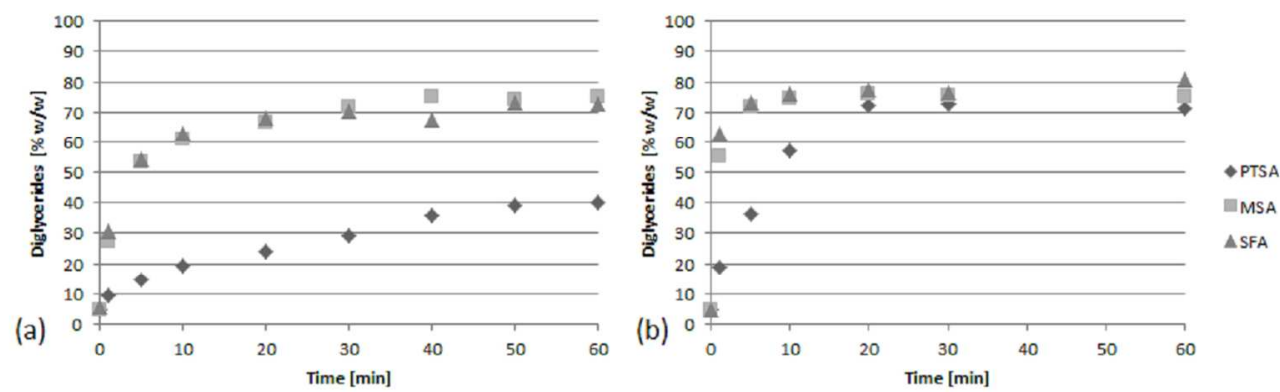

Fig. 6 DAG concentration versus time for solvent-assisted transesterification of MAG at (a) $35^{\circ} \mathrm{C}$ and (b) 70 ${ }^{\circ} \mathrm{C}$ (PTSA= $\mathrm{p}$-toluenesulfonic acid, MSA=methanesulfonic acid, SFA=sulfuric acid) $361 \times 114 \mathrm{~mm}$ (96 x 96 DPI) 
Page 23 of 26

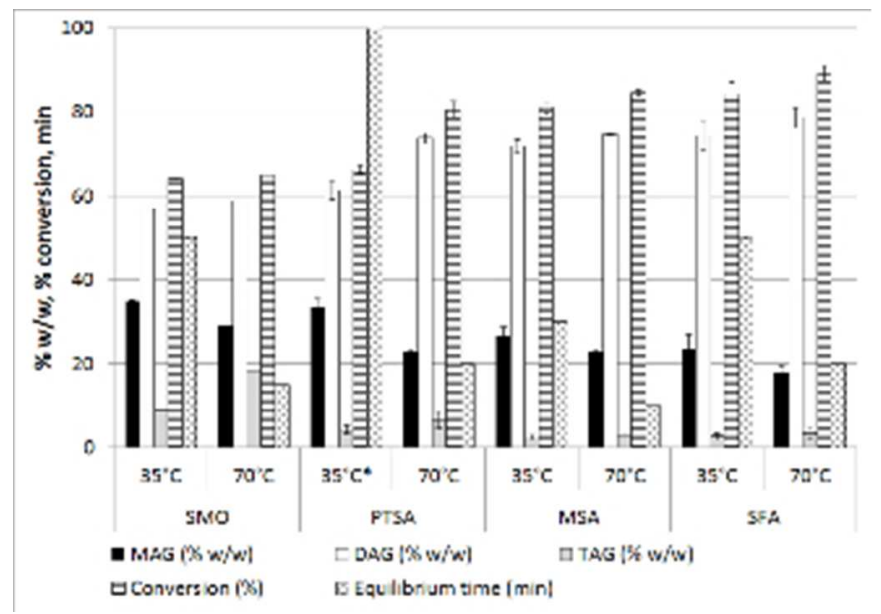

Fig. 7 Overview of the results for all solvent-assisted reactions ( $S M O=$ sodium methoxide, PTSA=ptoluenesulfonic acid, MSA=methanesulfonic acid, SFA=sulfuric acid), * for PTSA at $35^{\circ} \mathrm{C}$ reaction time was $300 \mathrm{~min}$ (equilibrium could not be reached) $87 \times 61 \mathrm{~mm}(96 \times 96 \mathrm{DPI})$ 


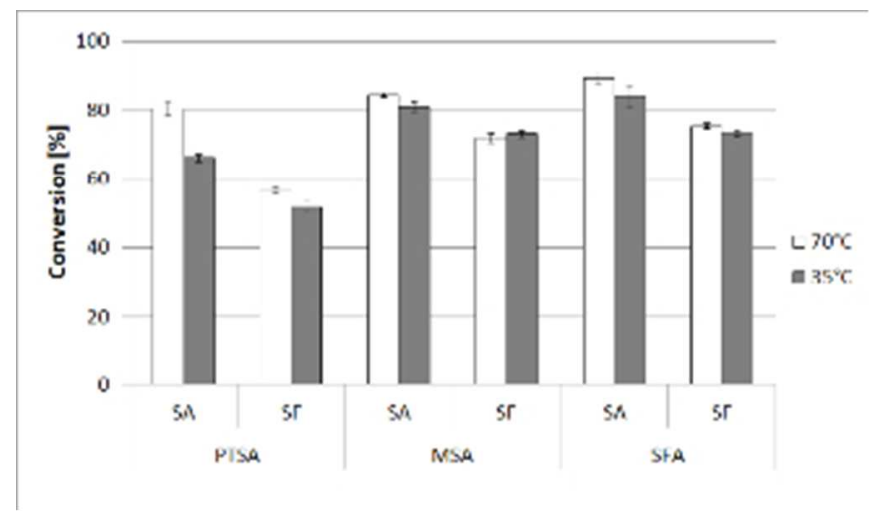

Fig. 8 Conversion for synthesis of DAG from MAG at $70^{\circ} \mathrm{C}$ and $35^{\circ} \mathrm{C}$ in solvent-assisted (SA) and solvent-free (SF) conditions using different acidic catalysts (PTSA = p-toluenesulfonic acid, MSA = methanesulfonic acid, SFA $=$ sulfuric acid), standard reaction time of $60 \mathrm{~min}$ (prolonged to $180 \mathrm{~min}$ for PTSA-SF in order to reached equilibrium, prolonged to $300 \mathrm{~min}$ for PTSA-SA without reaching equilibrium) $86 \times 50 \mathrm{~mm}(96 \times 96 \mathrm{DPI})$ 


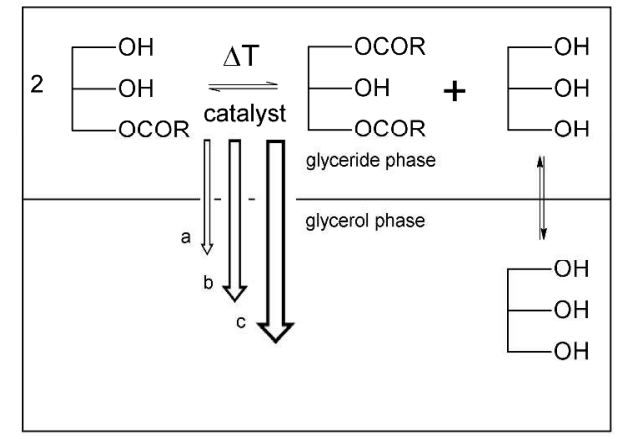

solvent-free reaction

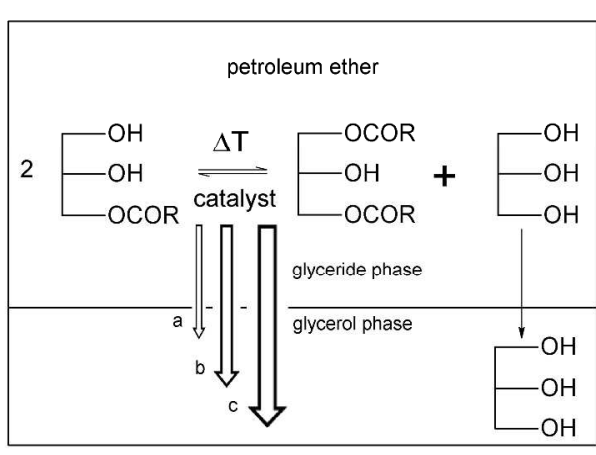

solvent-assisted reaction

Fig. 9 Schematic representation of catalyst movement to glycerol layer for solvent-free and solvent-assisted reactions with (a) p-toluenesulfonic acid, (b) methanesulfonic acid and (c) sulfuric acid. Line in the middle represents the phase separation.

$341 \times 133 \mathrm{~mm}(300 \times 300 \mathrm{DPI})$ 


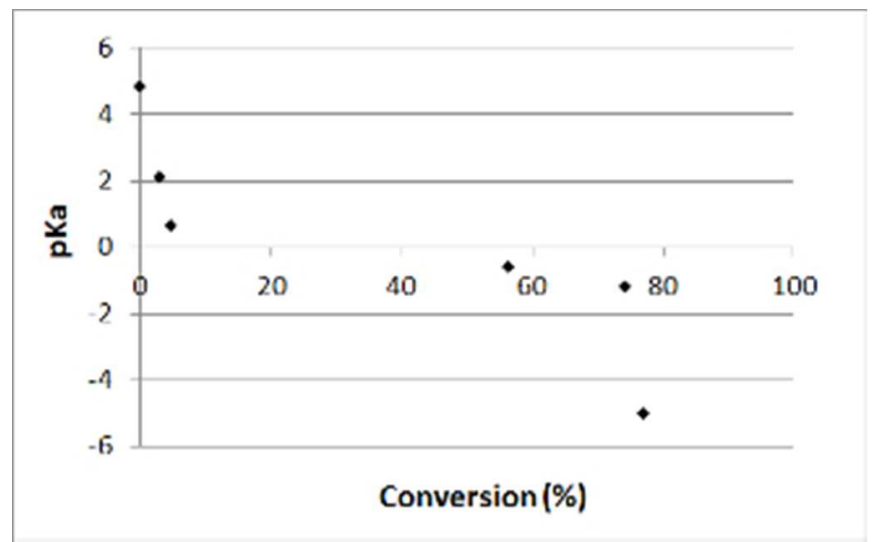

Fig. 10 Solvent-free acid-catalyzed transesterification of MAG using different acidic catalysts $\left(60 \mathrm{~min}, 70^{\circ} \mathrm{C}\right)$, pKa versus conversion (pKa values from reference 22) $86 \times 53 \mathrm{~mm}(96 \times 96 \mathrm{DPI})$ 